\title{
CHAOTIC DIFFERENCE EQUATIONS: GENERIC ASPECTS
}

\author{
BY
}

HANS-WILLI SIEGBERG

Dedicated to my father, Johannes Siegherg, 1915-1975.

\begin{abstract}
It is shown that in the set of all continuous selfmaps of a compact acyclic polyhedron (i.e. the homology groups of the space vanish in all dimensions $>0$ ) the chaotic maps form a dense set. The notion of chaos used here is that of $\mathrm{Li}$ and Yorke. If this notion is slightly weakened ("almost chaotic") the density result can be improved by the theorem that the set of almost chaotic (continuous) selfmaps of a compact acyclic polyhedron $P$ contains a residual subset of the space of all continuous selfmaps of $P$. Moreover, the topological entropy of such a generic almost chaotic map is shown to be infinite. The basic ingredients of the proofs are from fixed point index theory.
\end{abstract}

0. Introduction. In this paper we discuss the 'typical' behavior of difference equations

$$
x_{n+1}=f\left(x_{n}\right), \quad n \in N,
$$

where $f: X \rightarrow X$ is a continuous map of a compact polyhedron $X$ into itself. It is well known $[13,15,18]$ that if $X$ is a compact real interval $I$ and if $(0.1)$ has a periodic solution of period 3 (of period 5) then (0.1) has periodic solutions of all periods (resp. all periods $\neq 3$ ) as well as uncountably many aperiodic solutions, i.e. the difference equation (0.1) is chaotic, see Chapter 1.

In [11] it was shown that any continuous map of an interval $I$ into itself can be approximated (in the max-norm) by a continuous map which has a periodic point of period 3. In [7], see also [3, 17], it was proved that any continuous map of an interval $I$, which is sufficiently close to a given map with a periodic point of period 3 , has a periodic point of period 5. Hence, by Šarkovskii's theorem $[15,18]$ the set of (continuous) chaotic selfmaps of the inverval $I$ contains an open and dense subset of the space $C^{0}(I, I)$ of all continuous functions from $I$ into itself. Thus, chaotic behavior is a $C^{0}$-generic property for interval maps.

It is the purpose of this paper to prove that this is true, essentially, also for mappings of certain higher-dimensional spaces (acyclic polyhedra). Since the results described above are purely one-dimensional (their proofs rely heavily on the natural order of the real line) our proof requires tools different from the familiar techniques used in the context of interval mappings. (The basic ingredients of our proofs will be fixed point index and degree arguments.) In particular, appropriate conditions for

Received by the editors September 7, 1982.

1980 Mathematics Subject Classification. Primary 58F13, 58F22, 55M20. 
chaotic behavior in higher-dimensional spaces must be provided because the concept of snap-back repellers [14] is not suited for our purposes. To some extent, as a by-product of our techniques, it will turn out that $C^{0}$-generically, the topological entropy of a (chaotic) selfmap of a compact acyclic polyhedron, is infinite.

The paper is organized as follows. In Chapter 1 some preliminary results about chaos-generating mechanisms and topological entropy are provided. The main result of Chapter 2 is a general approximation theorem of mappings with nonvanishing fixed point index by chaotic mappings. This result is extended also to infinite dimensions. The genericity results are proved in Chapter 3.

ACKNOWLEDGEMENT. This paper is based on parts of the author's thesis at the University of Bremen advised by Heinz-Otto Peitgen. His influence over the past few years is gratefully acknowledged.

1. Preliminaries. The concept of chaotic behavior has experienced in recent time many specifications (e.g. sensitive dependence on initial conditions, Liapunov exponents, absolutely continuous invariant measures, etc.). The notion of chaos we use in this paper is the topological concept of $\mathrm{Li}$ and Yorke [13] which is defined as follows.

(1.1) Definition. Let $(X, d)$ be a metric space, $X^{\prime} \subset X$, and let $f: X^{\prime} \rightarrow X$ be a continuous map.

$f$ is called chaotic provided the following conditions are satisfied.

(1.1.1) $f$ has infinitely many periodic points with distinct periods.

(1.1.2) There is an uncountable subset $S \subset X^{\prime}$ (containing no periodic points) with the following properties.

(1) $\limsup _{n \rightarrow \infty} d\left(f^{n}(p), f^{n}(q)\right) \geqslant \alpha>0$ for all $p \neq q \in S$.

(2) $\lim \sup _{n \rightarrow \infty} d\left(f^{n}(p), f^{n}(a)\right) \geqslant \alpha>0$ for all $p \in S$ and for all periodic points $a$ of $f$.

(1.1.3) There is an uncountable subset $S_{0} \subset S$ with the property

$$
\liminf _{n \rightarrow \infty} d\left(f^{n}(p), f^{n}(q)\right)=0 \quad \text { for all } p, q \in S_{0} .
$$

If in Definition (1.1), condition (1.1.1) is replaced by

(1.1.4) For every $n \in N$ there is a periodic point with period $n$ for $f$, then we say that $f$ is chaotic in the sense of Li and Yorke.

We need two technical lemmas which are appropriate adaptations resp. generalizations of arguments developed in [13 and 5].

(1.2) Lemma. Let $A_{1}, A_{2} \subset R^{n}, n \geqslant 1$, be compact sets with $A_{1} \cap A_{2}=\varnothing$, and let $f$ : $A_{1} \cup A_{2} \rightarrow R^{n}$ be a continuous map.

(1.2.1) If $f\left(A_{1}\right) \supset A_{2}, f\left(A_{2}\right) \supset A_{1} \cup A_{2}$, then there exists an uncountable set $S \subset A_{1} \cup A_{2}$ satisfying (1.1.2).

(1.2.2) If $A_{1}, A_{2}$ are closed $n$-simplices (i.e. the convex closure of $n+1$ affinely independent points in $R^{n}$ ) and if $\left.f\right|_{A_{i}}$ is affine linear, $i=1,2$, with $f\left(A_{1}\right) \supset A_{2}$ and $f\left(A_{2}\right) \supset A_{1} \cup A_{2}$, then $f$ satisfies (1.1.4).

(1.2.3) If in (1.2.2) the stronger condition int $f\left(A_{1}\right) \supset A_{2}$, int $f\left(A_{2}\right) \supset A_{1} \cup A_{2}$ is satisfied, then $f$ is chaotic in the sense of Li and Yorke. 
Proof. Because the proof runs along lines similar to the proofs given in [13, Theorem 1] and in [14, Theorem 3.1] we give only a brief sketch.

Let $\Re$ be the set of sequences $M=\left\{M_{k}\right\}_{k \in N}$ where $M_{k}$ is either $A_{1}$ or $A_{2}$ with $M_{k}=A_{1} \Rightarrow k$ is square of an integer.

For each $r \in(0,1)$ choose $M^{r}=\left\{M_{k}^{r}\right\}_{k \in N}$ to be a sequence in $\mathbb{R}$ such that $\lim _{k \rightarrow \infty} P\left(M^{r}, k^{2}\right) / k=r$, where $P\left(M^{r}, k\right)$ is the number of $i$ 's in $\{1, \ldots, k\}$ for which $M_{i}^{r}=A_{1}$. For each $r \in(0,1)$ there is a point $x_{r}$ with $f^{k}\left(x_{r}\right) \in M_{k}^{r}$ for all $k$. Then $S:=\left\{x_{r} \mid r \in(0,1)\right\}$ satisfies (1.2.1).

If the conditions of (1.2.3) are satisfied then $f^{-k}\left(A_{2}\right)$ converges $(k \rightarrow \infty)$ to the (unique) fixed point of $f$ in $A_{2}$. To prove (1.2.3) the sequences $M^{r}, r \in(3 / 4,1)$, are further restricted using the sets $f^{-k}\left(A_{2}\right)$. This is done in a way analogous to the procedure described in [13 and 14].

In order to prove (1.2.2) observe the following fact. If $A \subset R^{n}$ is a closed $n$-simplex and if $g: A \rightarrow R^{n}$ is affine linear (or continuous and injective) with $g(A) \supset A$, then $g$ has a fixed point.

(1.3) REMARK. Lemma (1.2) which is sufficient for our purposes can be generalized in various ways, see e.g. $[14,17]$. For example, for $n=1$ the assumptions of (1.2) can be weakened as follows.

If $A_{1}, A_{2}$ are closed intervals such that $A_{1} \cap A_{2}$ is empty or a common boundary point and if $f: A_{1} \cup A_{2} \rightarrow R$ is continuous with $f\left(A_{1}\right) \supset A_{2}, f\left(A_{2}\right) \supset A_{1} \cup A_{2}$, then $f$ is chaotic in the sense of $\mathrm{Li}$ and Yorke.

Additional information which measures the complexity of behavior of a mapping in some sense quantitatively is provided by the topological entropy (see e.g. $[\mathbf{1}, \mathbf{4}]$ for a definition). The following lemma is useful for estimates of the topological entropy.

(1.4) Lemma. Let $(X, d)$ be a compact metric space, and let $A_{1}, \ldots, A_{n} \subset X$ be closed (nonempty) mutually disjoint subsets of $X$. Let $f: X \rightarrow X$ be continuous. Let $M=M\left(f ; A_{1}, \ldots, A_{n}\right)=\left(\left(a_{i j}\right)\right)$ be the matrix defined by the following rule:

$$
a_{i j}= \begin{cases}1, & f\left(A_{i}\right) \supset A_{j}, \\ 0, & \text { otherwise. }\end{cases}
$$

Then the topological entropy $h(f)$ of $f$ satisfies $h(f) \geqslant \log (\rho(M))$, where $\rho(M)$ denotes the spectral radius of the matrix $M$.

Proof (SKETCH). The lemma can be proved in a way very similar to the procedure in [5, Theorem 1] by applying techniques from symbolic dynamics. For sufficiently small $\varepsilon>0$ and for $k \geqslant 1$ we can find a $(k, \varepsilon)$-separated set [4] $Q_{k} \subset X$ with

$$
\text { card } Q_{k} \geqslant \operatorname{trace} M^{k} \text {. }
$$

Since $\lim \sup _{k \rightarrow \infty} k^{-1} \log \left(\operatorname{trace} M^{k}\right)=\log (\rho(M))$ [16, p. 149], the lemma follows from Bowen's definition of topological entropy [4].

(1.5) REMARK. If $f: X \rightarrow X$ is a continuous map of a compact metric space with $f\left(X_{1}\right) \cap f\left(X_{2}\right) \supset X_{1} \cup X_{2}$ for two (nonempty) disjoint compact subsets $X_{1}, X_{2} \subset X$, then by a result of Lasota and Yorke [12] there is a continuous ergodic measure 
invariant with respect to $f$. Thus, if the spectral radius of the matrix $M=$ $M\left(f ; A_{1}, \ldots, A_{n}\right)$ is bigger than one, then there is a continuous ergodic measure invariant with respect to some iterate $f^{k}$ of $f$, and therefore, there is a continuous ergodic measure on $X$ invariant with respect to $f$.

2. Density results. In all that follows below $P=(P, \tau)$ will denote a compact polyhedron with triangulation $\tau$; we always assume that $P$ has no isolated points and that the dimension of $P, \operatorname{dim} P$, is bigger than zero. For simplicity we assume, moreover, that $P$ is euclidean, i.e. $P$ is built up by closed simplices located in some fixed $R^{N}$. We call an open subset $U \subset P$ polyhedral if the closure $\bar{U}$ can be triangulated by $\tau \cap \bar{U}$.

The following proposition which is crucial for our proof of the main result $((2.2))$ of this chapter is a classical tool in fixed point index theory ("Hopf-construction" [10]).

(2.1) Proposition. Let $(P, \tau)$ be a compact polyhedron (not necessarily euclidean) and let $U \subset P$ be open and polyhedral. Let $f: \bar{U} \rightarrow P$ be continuous, and let $\varepsilon>0$. Then there exists a subdivision $\tau_{\varepsilon}$ of the triangulation $\tau$ and a subdivision $\tau_{\varepsilon}^{\prime}$ of $\tau_{\varepsilon} \cap \bar{U}$, and there is a simplicial mapping $f^{\prime}:\left(\bar{U}, \tau_{\varepsilon}^{\prime}\right) \rightarrow\left(P, \tau_{\varepsilon}\right)$ with the following properties.

(2.1.1) $d\left(f, f^{\prime}\right)<\varepsilon$.

(2.1.2) Any fixed point of $f^{\prime}$ is contained in the interior of some maximal simplex $\sigma \in \tau_{\varepsilon}^{\prime}$. (A simplex $\sigma$ in a triangulation $\tau$ is called maximal provided there is no simplex $\sigma_{1} \in \tau$ with $\sigma \subset \sigma_{1}$ and $\sigma \neq \sigma_{1}$.)

( $d$ is some metric which induces the topology of $P$.)

Proof. The proofs given in [10 and 6] apply to our situation.

We are now ready for the main result of this chapter. In the following we assume some familiarity with fixed point index resp. degree arguments, see e.g. [2,6,9].

(2.2) ThEOREM. Let $(P, \tau)$ be a compact polyhedron and let $U \subset P$ be open and polyhedral. Let $f: \bar{U} \rightarrow P$ be a continuous map with no fixed points on the boundary of $U$ such that the fixed point index $\operatorname{ind}(P, f, U) \neq 0$. Then for every $\varepsilon>0$ there is $a$ continuous map $g: \bar{U} \rightarrow P$ such that

(2.2.1) $d(f, g)<\varepsilon$,

(2.2.2) $\mathrm{g}$ is chaotic in the sense of Li and Yorke.

Proof. Let $f^{\prime}: \bar{U} \rightarrow P$ be a simplicial map obtained by (2.1). If $\varepsilon>0$ is sufficiently small then by the homotopy invariance of the fixed point index we have

$$
\operatorname{ind}\left(P, f^{\prime}, U\right)=\operatorname{ind}(P, f, U) \neq 0,
$$

and therefore, $f^{\prime}$ has a fixed point (which is contained in the interior of some maximal simplex of $\tau_{\varepsilon}^{\prime}$ ). Hence, since $f^{\prime}$ is a simplicial map, there is a maximal simplex $\sigma \in \tau_{\varepsilon}^{\prime}$ such that $f^{\prime}(\sigma) \supset \sigma, f^{\prime}(\sigma) \in \tau_{\varepsilon}$.

Now choose a triangulation $\tilde{\tau}$ of $\sigma$ satisfying the following properties.

(1) The boundary $\partial \sigma$ of $\sigma$ is not subdivided by the triangulation $\tilde{\tau}$.

(2) There are (at least) two simplices $\sigma_{1}, \sigma_{2} \in \tilde{\tau}$ with $\operatorname{dim} \sigma_{1}=\operatorname{dim} \sigma_{2}=\operatorname{dim} \sigma$, $\sigma_{1} \cap \sigma_{2}=\varnothing, \sigma_{i} \cap \partial \sigma=\varnothing, i=1,2$. 
(Such a triangulation $\tilde{\tau}$ is provided, for instance, by the $r$ th barycentric subdivision mod $\partial \sigma$ of $\sigma[6,10], r$ sufficiently large.)

In order to obtain $g$ we modify $f^{\prime}$ appropriately on $\sigma$. Let $T=\left(\tau_{\varepsilon}^{\prime} \backslash \sigma\right) \cup \tilde{\tau}$. Then we can find a simplicial map $g:(\bar{U}, T) \rightarrow\left(P, \tau_{\varepsilon}\right)$ with the following properties.

(a) $g_{\mid \partial \sigma}=f^{\prime}{ }_{\mid \partial \sigma}$.

(b) $\sigma_{1}$ and $\sigma_{2}$ are mapped onto $f^{\prime}(\sigma) \in \tau_{\varepsilon}$ by $g$.

(c) Every vertex in $\tilde{\tau}$ which is not contained in $\partial \sigma \cup \sigma_{1} \cup \sigma_{2}$ is mapped onto some vertex of $f^{\prime}(\sigma)$ by $g$.

(d) $g$ and $f^{\prime}$ coincide on $P \backslash \sigma$.

Because of (1.2) the map $g$ is chaotic in the sense of Li and Yorke, and, obviously, we have, assuming $\operatorname{mesh}\left(\tau_{\varepsilon}\right)<\varepsilon, d(f, g)<2 \varepsilon$.

(2.3) Remarks. (1) Theorem (2.2) is also valid for curvilinear polyhedra, i.e. spaces homeomorphic to euclidean polyhedra.

(2) Because of (1.4) we have that the topological entropy of $g$ in the theorem above satisfies $h(g) \geqslant \log 2$. By making the triangulation $\tilde{\tau}$ in the proof above sufficiently small we can achieve that $k, k \geqslant 2$ fixed, mutually disjoint simplices $\sigma_{i}, 1 \leqslant i \leqslant k$, of $\tilde{\tau}$ are mapped onto $f^{\prime}(\sigma)$ by $g$, with consequence $h(g) \geqslant \log k$.

(3) If the polyhedron $P$ is acyclic (see e.g. [6]) and if $U=P$ then $\operatorname{ind}(P, f, P)=$ $L(f)=1(L(f)=$ Lefschetz number of $f$, see e.g. [6]) and (2.2) applies. In particular, if $P$ is a closed $n$-simplex then chaotic mappings (in the sense of $\mathrm{Li}$ and Yorke) are dense in $C^{0}(P, P)$, and for $n=1$ we obtain again Kloeden's result in [11].

In order to extend (2.2) to infinite dimensions we need the following lemma which is an elementary consequence of (2.2) using 'standard' techniques.

(2.4) Lemma. Let $U \subset R^{n}$ be open and bounded, and let $f: \bar{U} \rightarrow R^{n}$ be a continuous map with no fixed points on the boundary of $U$ such that the (Brouwer) degree $\operatorname{deg}(\operatorname{Id}-f, U, 0) \neq 0$. Let $\varepsilon>0$. Then there is a point $x_{0} \in U$ such that for any $r>0$ there is a continuous map $g_{r}: \bar{U} \rightarrow R^{n}$ satisfying the following properties.

(2.4.1) $\left\|f-g_{r}\right\|<\varepsilon$.

(2.4.2) $g_{r}$ is chaotic in the sense of Li and Yorke.

(2.4.3) $g_{r}$ coincides with $f$ on $\bar{U} \backslash B\left(x_{0} ; r\right),\left(B\left(x_{0} ; r\right)=\left\{x \in R^{n} \mid\left\|x-x_{0}\right\| \leqslant r\right)\right.$.

Proof. Let $P \subset R^{n}$ be a closed $n$-simplex such that int $P \supset \bar{U} \cup f(\bar{U})$. Then $\operatorname{ind}(P, f, U)=\operatorname{deg}(\operatorname{Id}-f, U, 0)$ (see e.g. [9]) and Theorem (2.2) applies (we can assume that $U$ is polyhedral in some triangulation of $P$ ).

Let $\sigma \subset \bar{U}$ be the closed $n$-simplex obtained in the proof of (2.2); let $x_{0} \in$ int $\sigma$ and let $r>0$ be sufficiently small such that $B\left(x_{0} ; r\right) \subset$ int $\sigma$. If in the proof of (2.2) the triangulation $\tilde{\tau}$ is sufficiently small then the simplices $\sigma_{1}, \sigma_{2}$ can be chosen such that $\sigma_{1}, \sigma_{2} \subset$ int $B\left(x_{0} ; r\right)$. Let $g: \bar{U} \rightarrow R^{n}$ be the map constructed in (2.2) with $\sigma_{1}, \sigma_{2}$ chosen as described above. Now fix $s<r$ such that $\sigma_{1}, \sigma_{2} \subset B\left(x_{0} ; s\right)$, and let $\varphi$ : $\bar{U} \rightarrow[0,1]$ be a continuous function with

$$
\varphi(x)= \begin{cases}0, & x \in B\left(x_{0} ; s\right), \\ 1, & x \in \bar{U} \backslash B\left(x_{0} ; r\right) .\end{cases}
$$


Define $g_{r}: \bar{U} \rightarrow R^{n}$ by

$$
g_{r}(x):=\varphi(x) \cdot f(x)+(1-\varphi(x)) \cdot g(x),
$$

and the lemma follows $\left(\left\|f-g_{r}\right\|<2 \varepsilon\right)$.

(2.5) THEOREM. Let $E$ be a normed (infinite-dimensional) real or complex vector space, and let $U \subset E$ be open and bounded. Let $F: \bar{U} \rightarrow E$ be continuous and compact (i.e. $F(\bar{U})$ is relatively compact) with no fixed points on the boundary of $U$ such that the (Leray-Schauder) degree $\operatorname{deg}(\mathrm{Id}-F, U, 0) \neq 0$. Then for every $\varepsilon>0$ there is a continuous map $G: \bar{U} \rightarrow$ E such that

(2.5.1) $\|F-G\|<\varepsilon$,

(2.5.2) $G$ is chaotic in the sense of Li and Yorke.

Proof. Since $F$ is compact there exists a continuous map $F_{1}: \bar{U} \rightarrow E$ satisfying the following properties, see e.g. [2,9].

(a) $\left\|F-F_{1}\right\|<\varepsilon$.

(b) $F_{1}$ is compact, and $F_{1}(\bar{U})$ is contained in a finite-dimensional subspace $E_{1} \subset E$.

(c) $\operatorname{deg}(\mathrm{Id}-F, U, 0)=\operatorname{deg}\left(\mathrm{Id}-F_{1 \mid \bar{U} \cap E_{1}}, U \cap E_{1}, 0\right)(\varepsilon>0$ sufficiently small $)$.

Set $f=F_{1 \mid \bar{U} \cap E_{1}}: \bar{U} \cap E_{1} \rightarrow E_{1}$, and for $r>0$ let $g_{r}: \bar{U} \cap E_{1} \rightarrow E_{1}$ be the map obtained from (2.4). We choose $r>0$ sufficiently small such that the following conditions are satisfied.

(1) $B\left(x_{0} ; r\right)=\left\{x \in E \mid\left\|x-x_{0}\right\| \leqslant r\right\} \subset U$.

(2) $x \in B\left(x_{0} ; r\right) \Rightarrow\left\|F_{1}(x)-F_{1}\left(x_{0}\right)\right\|<\varepsilon$ (for $x_{0}$ see (2.4)).

By Dugundji's theorem [8] there is a retraction

$$
R: \bar{U} \rightarrow B\left(x_{0} ; r\right) \cap E_{1} \text {. }
$$

Now set $\tilde{G}:=\left(g_{r} \circ R\right): \bar{U} \rightarrow E_{1}$, and let $\psi: E \rightarrow[0,1]$ be a continuous function with

$$
\psi(x)= \begin{cases}0, & x \in B\left(x_{0} ; s\right), \\ 1, & x \in E \backslash B\left(x_{0} ; r\right)\end{cases}
$$

$(s<r$ chosen as in the proof of (2.4)). Define $G: \bar{U} \rightarrow E$ by

$$
G(x)=\psi(x) \cdot F_{1}(x)+(1-\psi(x)) \cdot \tilde{G}(x)
$$

and the theorem follows $(\|F-G\|<4 \varepsilon)$.

3. Stability results. As an immediate consequence of (2.2) and (1.3) we obtain that chaotic behavior (in the sense of $\mathrm{Li}$ and Yorke) is a $C^{0}$-generic property for interval maps.

(3.1) Theorem. Let $I=[a, b]$ be a compact real interval, and let $n \in N, n \geqslant 2$. Then there is an open and dense subset $E_{n}=E_{n}(I, I)$ of $C^{0}(I, I)$ such that for any $f \in E_{n}$ the following properties are satisfied.

(3.1.1) $f$ is chaotic in the sense of Li and Yorke.

(3.1.2) The topological entropy $h(f)$ satisfies $h(f) \geqslant \log n$.

(3.1.3) There is a continuous ergodic measure invariant with respect of $f$. 
Proof. Let $E_{n}(I, I)$ be the set of all continuous maps $f: I \rightarrow I$ satisfying the following condition. There are $n$ mutually disjoint subintervals $I_{1}, \ldots, I_{n}$ in int $I$ such that

$$
\bigcap_{j=1}^{n} \operatorname{int} f\left(I_{j}\right) \supset \bigcup_{j=1}^{n} I_{j} \text {. }
$$

Because of (1.2)-(1.4) every $f \in E_{n}$ satisfies (3.1.1) and (3.1.2); because of (1.5) every $f \in E_{n}$ satisfies (3.1.3). From (2.2) and (2.3) it follows that $E_{n}$ is dense in $C^{0}(I, I)$, and it is easy to verify that $E_{n}$ is also open in $C^{0}(I, I)$.

(3.2) REMARK. Observe that (3.1.1) is somewhat sharper than the result in [7]: in [7] it is proved only that there is an open and dense subset of $C^{0}(I, I)$ such that each map in this subset has a periodic point of period 5 .

(3.3) Corollary. There is a residual subset \& $\subset C^{0}(I, I)$ such that any $f \in \mathbb{E}$ satisfies (3.1.1), (3.1.3) and, moreover, $h(f)=\infty$.

Typical examples of mappings with infinite topological entropy are shifts over an infinite alphabet, see e.g. [1].

There is an analogous result on the circle.

(3.4) Theorem. Let $\mathrm{D} D=\left\{f \in C^{0}\left(S^{1}, S^{1}\right) \mid L(f)=1-\operatorname{deg} f \neq 0\right\}$ and let $n \in N$, $n \geqslant 2$. Then there exists an open and dense (in iㅣ) subset $E_{n}\left(S^{1}, S^{1}\right)$ of $C^{0}\left(S^{1}, S^{1}\right)$ satisfying (3.1.1)-(3.1.3).

In higher dimensions the situation is much more delicate. It is not clear if there is a complete $n$-dimensional analogue of Theorem (3.1) (where "open and dense" is replaced by "residual"). A partial $n$-dimensional analogue of (3.1) is proved in the following theorem.

(3.5) THEOREM. Let $(P, \tau)$ be a compact polyhedron which is acyclic. Then there is a residual subset $\mathfrak{E} \subset C^{0}(P, P)$ such that every $f \in \mathcal{E}$ is almost chaotic in the sense of $L i$ and Yorke, i.e. for $f \in \mathfrak{Q}$ the following properties are satisfied.

(3.5.1) For every $n \in N$ there is a periodic point of period $n$ for $f$.

(3.5.2) There is an uncountable subset $S \subset P$ (containing no periodic points) such that

(1) $\lim \sup _{n \rightarrow \infty} d\left(f^{n}(p), f^{n}(q)\right) \geqslant \alpha>0$ for all $p \neq q \in S$,

(2) $\lim \sup _{n \rightarrow \infty} d\left(f^{n}(p), f^{n}(a)\right) \geqslant \alpha>0$ for all $p \in S$ and for all periodic points $a$ of $f$.

(3.5.3) For every $k \in N$ there exists $n_{k}>0$ and an uncountable subset $S_{k} \subset S$ with $\liminf _{n \rightarrow \infty} d\left(f^{n}(p), f^{n}(q)\right) \leqslant n_{k}$ for all $p, q \in S_{k}$, and $\lim _{k \rightarrow \infty} n_{k}=0$.

Proof. Let $g: P \rightarrow P$ be a continuous map satisfying the following condition.

(*) There are subdivisions $\tau^{\prime}$ of the triangulation $\tau$ and $\tau^{\prime \prime}$ of $\tau^{\prime}$ such that

(a) $g$ is a simplicial map $\left(P, \tau^{\prime \prime}\right) \rightarrow\left(P, \tau^{\prime}\right)$,

(b) there are two maximal and disjoint simplices $\sigma_{1}, \sigma_{2} \in \tau^{\prime \prime}$ with $g\left(\sigma_{1}\right)=g\left(\sigma_{2}\right)$ $=: \sigma \in \tau^{\prime}$ and $\sigma_{1} \cup \sigma_{2} \subset$ int $\sigma$ (see proof of (2.2)). 
Fix $k \in N$. Since $g$ is affine linear on $\sigma_{1}$ and $\sigma_{2}$ there is a closed simplex $\Sigma \subset$ int $\sigma_{1}$ $\left(\operatorname{dim} \Sigma=\operatorname{dim} \sigma_{1}\right)$ such that

(i) $g^{k}$ maps $\Sigma$ affine linear onto $\sigma$,

(ii) $g^{k-1}$ maps $\Sigma$ affine linear onto $\sigma_{2}$, and

(iii) $g^{i}$ maps $\Sigma$ affine linear into int $\sigma_{1}, 0 \leqslant i \leqslant k-2\left(g^{0}=\right.$ Id).

For $k=1$ set $\Sigma=\sigma_{1}$.

Because of the homotopy invariance of the fixed point index we can find $\varepsilon_{k}(g)>0$ such that, for all $g^{\prime} \in C^{0}(P, P)$ with $d\left(g, g^{\prime}\right)<\varepsilon_{k}(g)$,

$$
\operatorname{ind}\left(P,\left(g^{\prime}\right)^{k} \text {,int } \Sigma\right)=\operatorname{ind}\left(P, g^{k} \text {,int } \Sigma\right) \neq 0 .
$$

The latter fixed point index is different from zero because of the linearity of $g^{k}$ on $\Sigma$. Thus, $\left(g^{\prime}\right)^{k}$ has a fixed point in $\Sigma$ which is a periodic point of period $k$ for $g^{\prime}$ provided $\varepsilon_{k}(g)$ is sufficiently small.

For $k=1$ we choose $\varepsilon_{k}(g)=\varepsilon_{1}(g)$ as follows. As above let $\varepsilon_{1}(g)>0$ be sufficiently small such that, for all $g^{\prime} \in C^{0}(P, P)$ with $d\left(g, g^{\prime}\right)<\varepsilon_{1}(g)$,

$$
\operatorname{ind}\left(P, g^{\prime} \text {, int } \Sigma\right)=\operatorname{ind}(P, g \text {, int } \Sigma) \neq 0 \text {. }
$$

Moreover, if $\varepsilon_{1}(g)$ is sufficiently small, e.g. $\varepsilon_{1}(g)<\frac{1}{2} \operatorname{dist}\left(\sigma_{1} \cup \sigma_{2}, \partial \sigma\right)$, then, for every $g^{\prime} \in C^{0}(P, P)$ with $d\left(g, g^{\prime}\right)<\varepsilon_{1}(g)$,

$$
g^{\prime}\left(\sigma_{1}\right) \cap g^{\prime}\left(\sigma_{2}\right) \supset \sigma_{1} \cup \sigma_{2} \text {. }
$$

(This follows with an elementary degree argument.)

Denote by $e_{k}$ the set of all maps $g^{\prime} \in C^{0}(P, P)$ for which there is a map $g \in C^{0}(P, P)$ satisfying (*) with $d\left(g, g^{\prime}\right)<\varepsilon_{k}(g)$; we can assume $\bigodot_{k+1} \subset \bigodot_{k}$ for $k \in N$. Because of (2.2) the sets $\bigodot_{k}, k \in N$, are open and dense in $C^{0}(P, P)$; hence, $\mathrm{e}=\bigcap_{k \in N} e_{k}$ is residual.

By construction every map $f \in \mathcal{C}$ satisfies (3.5.1) and (3.5.2).

In order to verify (3.5.3) we conclude as follows. For $g \in C^{0}(P, P)$, which satisfies (*), we can find a sequence of closed simplices $\left\{B_{i}\right\}_{i \in N}\left(\operatorname{dim} B_{i}=\operatorname{dim} \sigma_{1}\right.$, $i \in N$ ) such that

(1) $B_{1}=\sigma_{1}, B_{i} \subset$ int $\sigma_{1}$ for $i \geqslant 2$,

(2) $g\left(B_{i+1}\right)=B_{i}$ for $i \in N$,

(3) $B_{i+1} \subset B_{i}$ for $i \in N$, and $\lim _{i \rightarrow \infty} \operatorname{diam} B_{i}=0$.

If $\varepsilon_{k}(g)$ is sufficiently small then every $g^{\prime} \in C^{0}(P, P)$ with $d\left(g, g^{\prime}\right)<\varepsilon_{k}(g)$ satisfies

$$
\left(g^{\prime}\right)^{k}\left(B_{k}\right) \supset \sigma_{1} \text { and }\left(g^{\prime}\right)^{i}\left(B_{k}\right) \cap \sigma_{2}=\varnothing, \quad 0 \leqslant i \leqslant k-1 .
$$

Therefore (using essentially the same kind of arguments as in [13]), we can construct an uncountable subset $S_{k}$ which satisfies (3.5.2) such that, for $p, q \in S_{k}$,

$$
\liminf _{n \rightarrow \infty} d\left(\left(g^{\prime}\right)^{n}(p),\left(g^{\prime}\right)^{n}(q)\right) \leqslant \operatorname{diam} B_{k}=: n_{k},
$$

which proves the theorem.

(3.6) Remark. (1) As (2.2), Theorem (3.5) is also valid for curvilinear polyhedra.

(2) A question left unsolved is whether (3.5) can be sharpened in such a way that every $f \in C$ is chaotic in the sense of $\mathrm{Li}$ and Yorke.

We conclude this chapter with a theorem which completes, in some sense, Theorem (3.5). 
(3.7) TheORem. Let $(P, \tau)$ be a compact polyhedron which is acyclic, and let $n \in N$, $n \geqslant 2$. Then there is an open and dense subset $E_{n}=E_{n}(P, P)$ of $C^{0}(P, P)$ such that for any $f \in E_{n}$ the following properties are satisfied.

(3.7.1) $h(f) \geqslant \log n$.

(3.7.2) There is a continuous ergodic measure invariant with respect to $f$.

Proof. This follows immediately (using similar arguments as in (3.5), see definition of $\left.\varepsilon_{1}(g)\right)$ from (2.2), (2.3), (1.4) and (1.5).

\section{REFERENCES}

1. R. L. Adler, A. G. Konheim and M. H. McAndrew, Topological entropy, Trans. Amer. Math. Soc. 114 (1965), 309-319.

2. H. Amann, Lectures on some fixed point theorems, Monograf. Mat., Inst. Mat. Pura Apl., Rio de Janeiro, 1974.

3. L. Block, Stability of periodic orbits in the theorem of Šarkovskii, Proc. Amer. Math. Soc. 81 (1981), $333-336$.

4. R. Bowen, Entropy for group endomorphisms and homogeneous spaces, Trans. Amer. Math. Soc. 153 (1971), 401-414.

5. R. Bowen and J. Franks, The periodic points of maps of the disk and the interval, Topology 15 (1976), $337-342$.

6. R. F. Brown, The Lefschetz fixed point theorem, Scott, Foresman \& Co., Glenview, Ill., 1971.

7. G. J. Butler and G. Pianigiani, Periodic points and chaotic functions in the unit interval, Bull. Austral. Math. Soc. 18 (1978), 255-265.

8. J. Dugundji, An extension of Tietze's theorem, Pacific J. Math. 1 (1951), 353-367.

9. G. Eisenack and C. Fenske, Fixpunkttheorie, Bibliographisches Institut, Mannheim, 1978.

10. H. Hopf, Über die algebraische Anzahl von Fixpunkten, Math. Z. 29 (1929), 493-524.

11. P. E. Kloeden, Chaotic difference equations are dense, Bull. Austral. Math. Soc. 15 (1976), 371-379.

12. A. Lasota and J. A. Yorke, On the existence of invariant measures for transformations with strictly turbulent trajectories, Bull. Acad. Polon. Sci. 25 (1977), 233-238.

13. T. Y. Li and J. A. Yorke, Period three implies chaos, Amer. Math. Monthly 82 (1975), 985-992.

14. F. R. Marotto, Snap-back repellers imply chaos in $R^{n}$, J. Math. Anal. Appl. 63 (1978), 199-223.

15. A. N. Šarkovskii, Coexistence of cycles of a continuous map of a line into itself, Ukrain. Mat. Ž. 16 (1964), 61-71.

16. M. Shub, Stabilité globale des systèmes dynamiques, Astérisque, vol. 56, Société Mathématique de France, Paris, 1978; $2^{\mathrm{e}}$ édition corrigée, 1980.

17. H. W. Siegberg, Zur komplexen Dynamik iterierter Abbildungen, Dissertation, University of Bremen, 1982.

18. P. Stefan, $A$ theorem of $\check{S}$ arkovskii on the existence of periodic orbits of continuous endomorphisms of the real line, Comm. Math. Phys. 54 (1977), 237-248.

Fachbereich Mathematik, University of Bremen, D-2800 Bremen 33, Federal Republic of GERMANY 